\title{
PRURITO VULVAR Y ENFERMEDAD DE FOX - FORDYCE, UN REPORTE DE CASO
}

\author{
FOX - FORDYCE DISEASE, A CASE REPORT \\ Katherine Luisa Contreras-Gala1,a
}

\begin{abstract}
RESUMEN
Se presenta un caso infrecuente de enfermedad de Fox-Fordyce en región vulvar, una dermatosis inflamatoria no infecciosa, infrecuente, de glándulas apocrinas, caracterizada por una erupción papular pruriginosa que involucra vulva, axila y región perianal, a predominio del sexo femenino, del cual no hay reportes nacionales. Se presenta una paciente de 53 años de edad, evaluada en consulta de ginecobstetricia, por presentar desde hace 5 meses, erupción papular en zona vulvar, asociado a prurito intermitente. Se tomó una biopsia de la lesión, y se envió al servicio de anatomía patológica, recibiéndose un fragmento de tejido de color pardo oscuro, de 0,3 ×0,2 ×0,1cm, en la cual se evidenció glándulas apocrinas dilatadas, con secreción espesa constituida por mucina, en su luz. Cumpliendo con los criterios histopatológicos, se estableció el diagnóstico de Enfermedad de Fox - Fordyce. Se consideró importante la presentación de este caso debido a la naturaleza infrecuente de esta enfermedad.
\end{abstract}

Palabras clave: Enfermedad de Fox-Fordyce; Prurito vulvar; Enfermedades de las glándulas sudoríparas; glándulas sudoríparas (fuente: DeCS BIREME).

\begin{abstract}
Fox-Fordyce disease is a non-infectious, infrequent inflammatory dermatosis of apocrine glands, isolated by a pruritic papular rash that usually begins frequently at puberty, and which may involve vulva, armpit and perianal region. It affects women more frequently, approximately in a 9 to 1 ratio, compared to men. In the presentation of the case of a female patient, 53 years old, in consultation of Gynecology-Obstetrics, of the General Maria Auxiliadora Hospital, for presenting for 5 months, papular eruption in vulvar area, associated with intermittent pruritus. A biopsy of the lesion was requested, and it was sent to the Pathological Anatomy Service, receiving a fragment of dark brown tissue, $0.3 \times 0.2 \times 0.1 \mathrm{~cm}$, which was automatically processed, obtaining a histological sheet, in which evidence dilated apocrine glands, which show a thick secretion made up of mucin, in its light. The patient met the histopathological criteria for the diagnosis of Fox - Fordyce disease. Consider the case presentation because of the infrequent nature of this disease.
\end{abstract}

Key words: Fox-Fordyce Disease; Pruritus vulvae; Sweat gland diseases; Sweat glands (source: MeSH NLM).

\section{TRADUCCIÓN}

La enfermedad de Fox-Fordyce fue descrita por primera vez en 1902 por George Henry Fox y John Addison Fordyce, es una dermatosis inflamatoria no infecciosa, infrecuente, de glándulas apocrinas ${ }^{(1)}$. Se caracteriza por ser de curso crónico y por manifestarse con pápulas del color de la piel, muy pruriginosas, en partes del cuerpo donde se concentran las glándulas apocrinas $^{(2)}$. Su etiopatogenia es poco conocida y afecta las áreas que poseen glándulas apocrinas: las axilas, las ingles, la región púbica, el periné, los labios mayores, las areolas y el ombligo. Aparece principalmente en mujeres pospuberales, entre los 13 ylos 35 años deedad, aunque se han comunicadocasos prepuberales y tras la menopausia ${ }^{(3,4)}$; sin predilección racial y con incidencia aún no establecida ${ }^{(2)}$. Afecta principalmente a mujeres en una relación de 9 a 1; de

${ }^{1}$ Hospital General María Auxiliadora, Lima - Perú.

a Médico Residente de Anatomía Patológica.

Citar como: Katherine Luisa Contreras-Gala. Prurito vulvar y enfermedad de fox-fordyce, un reporte de caso. Rev. Fac. Med. Hum. Abril 2020; 20(2):319321. DOI 10.25176/RFMH.v20i2.2516

Artículo publicado por la Revista de la Facultad de Medicina Humana de la Universidad Ricardo Palma. Es un artículo de acceso abierto, distribuído bajo los términos de la Licencia Creative Commons: Creative Commons Attribution 4.0 International, CC BY 4.0 (https://creativecommons.org/licenses/by/4.0/), que permite el uso no comercial, distribución y reproducción en cualquier medio, siempre que la obra original sea debidamente citada. Para uso comercial, por favor póngase en contacto con revista.medicina@urp.pe 
forma ocasional se ha descrito algún caso en varones ${ }^{(1)}$ Con la estimulación emocional o física de las glándulas apocrinas se observan exacerbaciones paroxísticas. Su tratamiento es discutido, se han utilizado distintos regímenes terapéuticos con resultados dispares y no siempre satisfactorios ${ }^{(3,4,5)}$.

La causa es una obstrucción intraepidérmica del conducto de las glándulas apocrinas, y se plantea la hipótesis que está determinada por factores hormonales y genéticos, con criterios histopatológicos que incluyen hiperqueratosis ${ }^{(2)}$, obstrucción del infundíbulo folicular ${ }^{(2)}$, cambios inflamatorios crónicos en la dermis ${ }^{(1)}$, y dilatación de los acinos de las glándulas apocrinas $^{(1)}$, las cuales muestran una secreción espesa de mucina eosinófila en su lumen ${ }^{(1)}$. El tratamiento no es sencillo, pues algunos esquemas terapéuticos pueden tener molestos efectos secundarios y las recidivas son frecuentes $^{(2)}$. En otras revisiones, no se especifica un tratamiento definitivo(1); pero se describen algunas opciones de tratamiento como los anticonceptivos orales combinados para tratar y disminuir los síntomas de mujeres en edad fértil(1). Inclusive la isotretinoína ha sido utilizada alguna vez en el tratamiento de esta condición ${ }^{(2)}$, con resultados variables.

El presente trabajo tiene como objetivo presentar un caso clínico de Enfermedad de Fox - Fordyce. Se decidió la presentación del caso por lo infrecuente de esta enfermedad.

\section{PRESENTACIÓN DEL CASO}

Se presenta el caso de una paciente de sexo femenino, de 53 años de edad, post menopaúsica con fecha de última regla hace cinco años y Papanicolaou negativo el 2019, la cual fue evaluada en consulta de ginecobstetricia, del hospital general María Auxiliadora, en noviembre del 2019, por presentar desde hace 5 meses, erupción papular en zona vulvar, asociado a prurito intermitente. Se tomó una biopsia de la lesión, y se envió al servicio de anatomía patológica, recibiéndose un fragmento de tejido de color pardo oscuro, de 0,3 x 0,2 x 0,1cm, el cual fue procesado adecuadamente, obteniéndose una lámina histológica, en la cual se evidenció glándulas apocrinas dilatadas, las cuales mostraban una secreción espesa constituida por mucina, en su luz; asociado a cambios inflamatorios leves en dermis (Figuras 1 y 2). La paciente cumplía criterios histopatológicos para el diagnóstico de Enfermedad de Fox - Fordyce.

Se diagnosticó como Enfermedad de Fox-Fordyce, debido a que ninguna medicación ninguna medicación ha demostrado ser convincentemente eficaz, se decidió mantener al paciente con tratamiento sintomático.

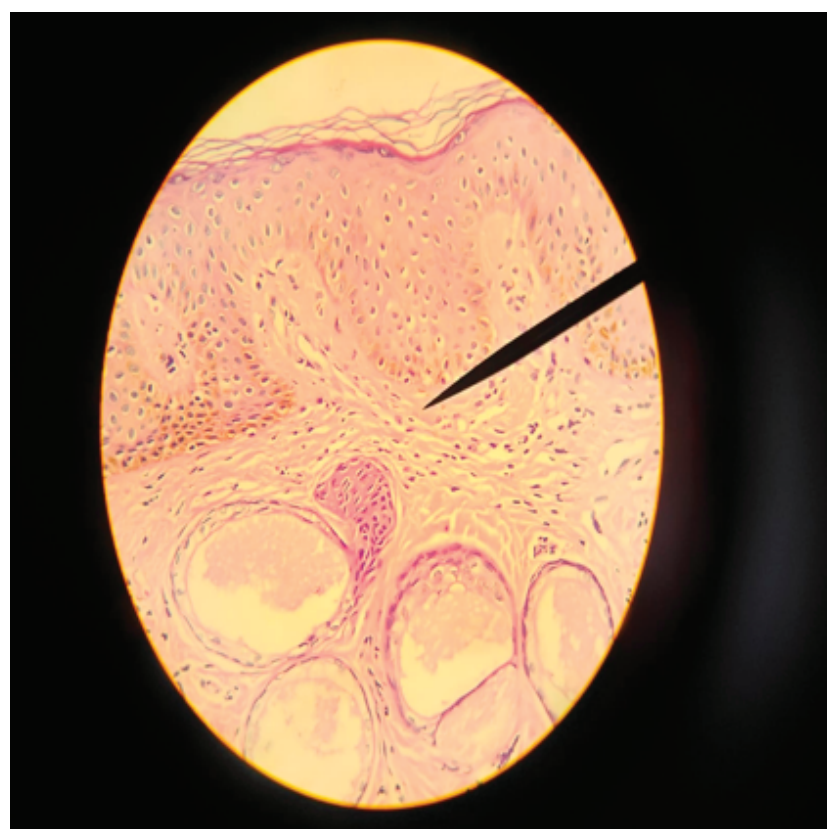

Figura 1. Cambio Inflamatorio leve en dermis (H-E 40x).

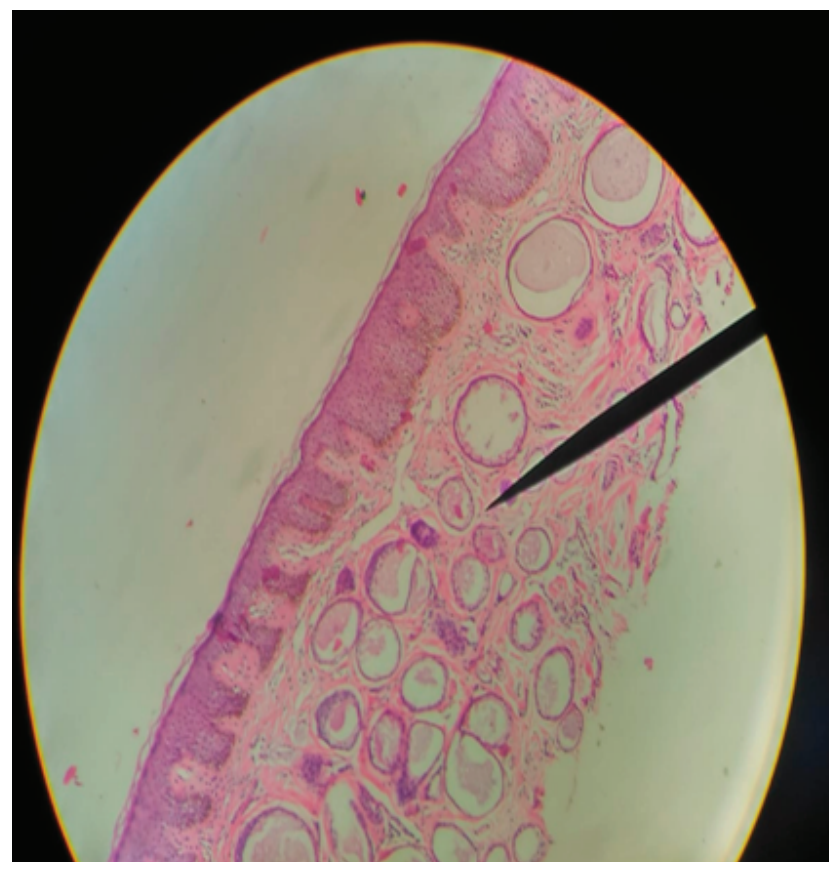

Figura 2. Presencia de glándulas apocrinas con secreción de mucina en su luz (H-E 10x).

\section{DISCUSIÓN}

En la biopsia de la lesión se evidenció glándulas apocrinas dilatadas, las cuales mostraban una secreción espesa de mucina eosinófila en su luz. Dentro de los criterios histopatológicos para el diagnóstico de la Enfermedad de Fox-Fordyce, se incluyen hiperqueratosis ${ }^{(2)}$, obstrucción del infundíbulo folicular(2), cambios inflamatorios crónicos en la dermis $^{(1)}$, y dilatación de los acinos de las glándulas apocrinas $^{(1)}$, las cuales muestran una secreción espesa eosinófila de mucina en su lumen ${ }^{(1)}$. El presente caso cumple con dos de los cuatro criterios mencionados, 
los cuales asociados a las características clínicas de la paciente, nos permitió realizar el diagnóstico final de Enfermedad de Fox-Fordyce, descartando diagnósticos diferenciales como liquen amiloide, liquen nitidus, siringomas eruptivos, foliculitis infecciosa, mucinosis folicular y enfermedad de Darier, entre otros ${ }^{(6,7)}$.

La etiología no ha sido aún esclarecida. No se han descrito alteraciones genéticas ni polimorfismos asociados ${ }^{(3)}$; sin embargo, Rubio y colaboradores involucran elementos genéticos, endocrinos, metabólicos o ambientales sin que exista evidencia a favor de ninguno de ellos ${ }^{(3,4,5)}$.

Como opción terapéutica de primera línea, algunos autores proponen el uso de inhibidores de la calcineurina, específicamente pimecrolimus, ya que este medicamento fue desarrollado para tratar procesos inflamatorios como los que caracterizan la enfermedad luego de la ruptura del conducto glandular y también porque, mediante un mecanismo desconocido, inhibe la hiperqueratosis y la obstrucción folicular ${ }^{(8)}$. No obstante, en otras literaturas no se especifica un tratamiento definitivo(1); pero se describen algunas opciones de tratamiento como los anticonceptivos orales combinados para tratar y disminuir los síntomas de mujeres en edad fértil(1), lo cual actualmente, junto con tratamiento sintomático, es lo que se plantea iniciar como tratamiento en la paciente. La paciente recibió únicamente tratamiento sintomático, siendo esta una limitación en la evolución del cuadro clínico.

\section{CONCLUSIÓN}

Es de suma importancia que en casos de prurito vulvar de etiología incierta, y al encontrarnos frente a una biopsia de vulva en dichos casos, tengamos en cuenta el diagnóstico presentado en este reporte de caso. Por medio del presente artículo, se buscó reportar el primer caso a nivel nacional de Enfermedad de Fox- Fordyce en vulva.

Agradecimientos: A la Doctora Sigrid Abril Santos Laurente, por sus enseñanzas y orientación, en este caso.

Contribuciones de autoría: La autora participó en la génesis de la idea, recolección e interpretación de datos, análisis de resultados y preparación del manuscrito del presente artículo de investigación.

Financiamiento: Autofinanciado.

Conflicto de interés: La autora declara no tener conflicto de interés en la publicación de este artículo.

Recibido: 08 de diciembre 2019

Aprobado: 17 de marzo 2020

\section{Correspondencia: Katherine Luisa Contreras Gala.}

Dirección:Jr. Trinidad Morán 275. Urbanización El Retablo-IEtapa, Comas, Lima-Perú. Teléfono: 979483818

Correo:katyc19@hotmail.com

\section{REFERENCIAS BIBLIOGRÁFICAS}

1. Kurman RJ, Ellenson LH, Ronnet BM. Blaustein's pathology of the female genita tract. Sixth edition, Springer

2. Echauri Anahí, Cubilla Elisa, Guzmán Antonio. Enfermedad de Fox-Fordyce: Caso clínico y revisión de la literatura. DermatologíaCMQ2013;11(1):26-28.

3. Rubio C, Mayor M, Marlin MA, González MJ, Contreras F, Gasado M. Enfermedad de Fox Fordyce. Actas Dermosifiliogr. 2004;95:314-6.

4. Consulich Claudia L, Meik S, Vicente AM, Abeldaño A. Pápulas foliculares pruriginosas. Dermatol. 2012;18(5):405-08.
5. García Arpa M, Sánchez Gami Nero P, Vera Iglesias E, Marlin Dávila F. Múltiples pápulas vulvares. Acta Dermosifiliogr. 2007;98(07):499-500.

6. Martínez Luna E, Rebollo Domínguez N, Vega Memije M, Arenas R. Siringomas vulvares: Informe de dos casos. Ginecol Obstet Mex. 2006;74:273-6.

7. Kao PH, Hsu ChK, Lee JY."Clinicopathological Study of Fox-Fordyce disease". Journal of Dermatology 2009; 36: 485-490.Kao PH, Hsu ChK, Lee JY. "Clinicopathological Study of Fox-Fordyce disease". Journal of Dermatology 2009; 36: 485-490.

8. Chandrakumar A, Francis N, Morar N. "Efficacy of Pimecrolimus in Fox-Fordyce Disease". Open Dermatology Journal 2010; 4: 59-61. 